\title{
ROBUSTNESS-STRENGTH PERFORMANCE OF HIERARCHICAL ALPHA-HELICAL PROTEIN FILAMENTS
}

\author{
ZHAO QIN ${ }^{*, \dagger}$, STEVEN CRANFORD ${ }^{*, \dagger}$, \\ THEODOR ACKBAROW ${ }^{*, \ddagger}$ and MARKUS J BUEHLER ${ }^{*, \dagger, \S, \uparrow}$ \\ ${ }^{*}$ Laboratory for Atomistic and Molecular Mechanics \\ Department of Civil and Environmental Engineering \\ Massachusetts Institute of Technology \\ 77 Massachusetts Ave., Room 1-235A\&B \\ Cambridge, MA, USA \\ ${ }^{\dagger}$ Center for Materials Science and Engineering \\ Massachusetts Institute of Technology \\ 77 Massachusetts Ave., Cambridge, MA, USA \\ ${ }^{\ddagger}$ Max Planck Institute of Colloids and Interfaces \\ Theory Division, Science Park Golm \\ 14424 Potsdam, Germany \\ $\S_{\text {Center for Computational Engineering }}$ \\ Massachusetts Institute of Technology \\ 77 Massachusetts Ave., Cambridge, MA, USA \\ ףmbuehler@MIT.EDU
}

Received 3 December 2009

Accepted 13 February 2009

\begin{abstract}
An abundant trait of biological protein materials are hierarchical nanostructures, ranging through atomistic, molecular to macroscopic scales. By utilizing the recently developed Hierarchical Bell Model, here we show that the use of hierarchical structures leads to an extended physical dimension in the material design space that resolves the conflict between disparate material properties such as strength and robustness, a limitation faced by many synthetic materials. We report materiomics studies in which we combine a large number of alpha-helical elements in all possible hierarchical combinations and measure their performance in the strength-robustness space while keeping the total material use constant. We find that for a large number of constitutive elements, most random structural combinations of elements $(>98 \%)$ lead to either high strength or high robustness, reflecting the so-called banana-curve performance in which strength and robustness are mutually exclusive properties. This banana-curve type behavior is common to most engineered materials. In contrast, for few, very specific types of combinations of the elements in hierarchies $(<2 \%)$ it is possible to maintain high strength at high robustness levels. This behavior is reminiscent of naturally observed material performance in biological materials, suggesting that the existence of particular hierarchical structures facilitates a fundamental change of the material performance. The results suggest that biological materials may have developed under evolutionary pressure to yield materials with multiple objectives, such as high strength and high robustness, a trait that can be achieved by
\end{abstract}

๑Corresponding author. 


\begin{abstract}
utilization of hierarchical structures. Our results indicate that both the formation of hierarchies and the assembly of specific hierarchical structures play a crucial role in achieving these mechanical traits. Our findings may enable the development of self-assembled de novo bioinspired nanomaterials based on peptide and protein building blocks.
\end{abstract}

Keywords: Mechanical properties; nanomaterials; peptide nanostructure; rupture mechanics; fracture; alpha-helix; hierarchies; Bell model; materiomics; strength; robustness; nanomechanics.

PACS: 87.14.Ee, 82.37.Rs, 46.50.+a, 62.20.-x, 61.46.-w, 81.40.Np

\title{
1. Introduction
}

The origin of how naturally occurring biological protein materials (e.g. cellular protein filaments, spider silk, bone, tendon, skin) are capable of unifying disparate mechanical properties such as strength (ability to sustain large stresses without fracture) and robustness (ability to undergo deformation without fracture, despite the presence of defects, equivalent to toughness) is of significant interest. However, the molecular basis of these properties remains largely unknown [Buehler and Yung, 2009; Fratzl and Weinkamer, 2007; Ingber, 2006; Langer and Tirrell, 2004]. Many synthetic materials are not capable of unifying strength and robustness, being either extremely strong with little ductility (e.g. ceramics, glass, silicon), or weak with extreme ductility (e.g. soft metals like copper or gold) [Hirth and Lothe, 1982]. Figure 1 displays the mechanical behavior of engineered materials (Fig. 1(a)) and biological materials (Fig. 1(b)), clearly illustrating the distinct mechanical performance [Ashby et al., 1995; Fratzl et al., 2004]. The combination of these disparate properties into synthetic materials remains an open challenge on the way towards the development of biomimetic structures and material designed from the nanoscale up.

Here we analyze the mechanical performance of biological protein materials using a bottom-up approach, exemplified for alpha-helical (AH) protein domains as model systems. By utilizing the recently developed Hierarchical Bell Model [Ackbarow and Buehler, 2009; Buehler et al., 2008], we show that the use of hierarchical structures leads to an extended physical dimension in the material design space that resolves the conflict between disparate material properties such as strength and robustness, a limitation faced by many synthetic materials. AHs are universally found nanostructural components of many biological protein materials. These protein domains play a crucial role in the signaling and deformation behavior of cytoskeletal protein networks in cells (e.g. intermediate filaments vimentin and lamin as well as actin [Alberts et al., 2002; Herrmann and Aebi, 2004; Rowat et al., 2008], and in determining the mechanical properties of hair, hoof, feather and many other important structural protein materials [Alberts et al., 2002]. In these materials, nanostructured $\mathrm{AH}$ based protein domains universally define their nanoscale architecture.

The folded structure of proteins is stabilized by a variety of chemical driving forces including hydrophobic effects, H-bond formation as well as charge interactions 


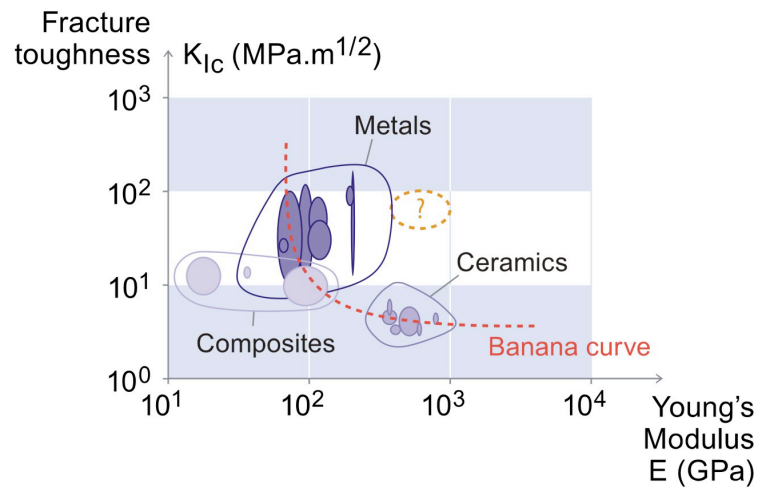

(a)

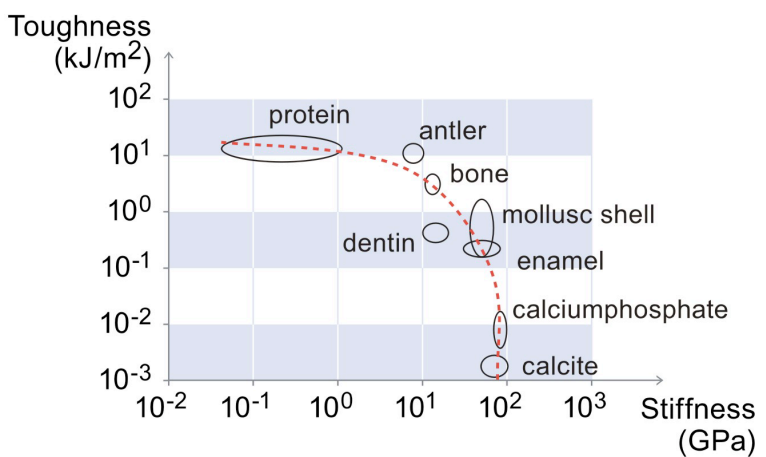

(b)

Fig. 1. Performance of engineered and biological materials in the stiffness-toughness domain. Subplot (a) depicts toughness and stiffness values for synthetic materials, such as metals, alloys and ceramics (plot adapted from Ashby et al. [1995]). Subplot (b) compares the toughness and stiffness properties for a number of biological materials (plot adapted from Fratzl et al. [2004]). Biological composites, such as antler, dentin, bone and enamel are result of a "reinforcing" combination of the protein toughness and the mineral-stiffness. Remarkably, all these materials lie - in contrast to biological materials — on a so-called "banana-curve", an inverse relation between increasing toughness an decreasing stiffness. The yellow region shows the property region of high toughness and stiffness that may be accessible through creating bio-inspired materials.

[Murphy, 2001]. An AH is generated when a single polypeptide chain twists around on itself, stabilized by $\mathrm{H}$-bonds made between every fourth residue, linking the $\mathrm{O}$ backbone atom (hydrogen acceptor, providing free electrons) of peptide $I$ to the $\mathrm{N}$ backbone atom (hydrogen donor) of peptide $i+4$ in the polypeptide chain. Consequently, at each convolution, 3-4 H-bonds are found in a parallel arrangement that stabilize the helical configuration of AHs [Alberts et al., 2002]. Due to the stabilizing role of H-bonds [Murphy, 2001], rupture of these protein structures itself is mainly determined by breaking of these $\mathrm{H}$-bonds, which is in particular the case when the breaking of $\mathrm{H}$-bonds occurs in a highly driven system. Once these H-bonds are 
broken, the protein has the degrees of freedom necessary for protein unfolding. In contrast, at very small deformation rates near the equilibrium the H-bond rupture needs significantly less force than the protein unfolding itself, which is determined by entropic effects of stretching the protein backbone (and restricting the degrees of freedom again) (see, e.g. the discussion recently reported in Keten and Buehler [2008a]). In this article we focus on protein rupture mechanics in a highly driven system, and thus consider H-bonded interactions as the main contributor to determine their strength.

Through the analysis reported here, we illustrate that the nanostructural arrangement of universal protein building blocks plays a crucial role in defining their material performance. Thereby, the occurrence of material hierarchies (that is, the arrangement of subunits to units, which themselves form larger-scale structures, etc.) is a particularly important aspect. The analysis reported here is focused on $\mathrm{AH}$ based protein filaments to illustrate fundamental material concepts, with H-bonds representing the lowest level of a hierarchical unit. Such concepts could be extended and translated to the design of other nanostructures with a multitude of hierarchies not limited to physical bonds, such as hierarchically arranged carbon nano-tubes (CNT) or nanowire bundles [Qin et al., 2007].

\subsection{Hierarchical $\mathbf{H}$-bond protein structures}

In biological tissues, macroscopically applied stress is forwarded to micro-/ nanoscopic hierarchical scales, where rupture of H-bonds mediates deformation, thereby controlling the response at the macroscopic protein filament level. Since rupture of individual H-bonds equals a chemical reaction (breaking of the backbone $\mathrm{H}$-bond and the formation of H-bonds with surrounding water molecules governed by a difference in H-bond building energy), an integrated chemomechanical approach is compulsory for the understanding of protein fracture mechanisms and the development of constitutive mathematical relations.

The lowest hierarchy of AHs (and other protein structures) is typically composed of arrangements of chemically weak H-bond interactions, organizing amino acids in stable elementary building blocks such as AHs, which form hierarchical arrangements such as coiled-coils (CCs), supercoils and filamentous structures [Ackbarow and Buehler 2008; Alberts et al., 2002; Herrmann and Aebi, 2004; Rowat et al., 2008], exemplified for AH based structures as they appear in intermediate filaments [Herrmann and Aebi, 2004] (see Fig. 2). The key to enable the development of an accurate bottom-up mechanistic understanding of the strength properties of such $\mathrm{AH}$ protein domains and assemblies thereof must therefore include an explicit description of nanopatterned H-bond arrangements, by adapting a system view of materials, in the spirit of a merger of nano-structure and materials. The concept of hierarchical arrangements, where each element consists of several subelements provides a broadly applicable, yet simple model to describe the geometry of AH protein structures [Ahl and Allen, 1996]. The schematic representation depicted 


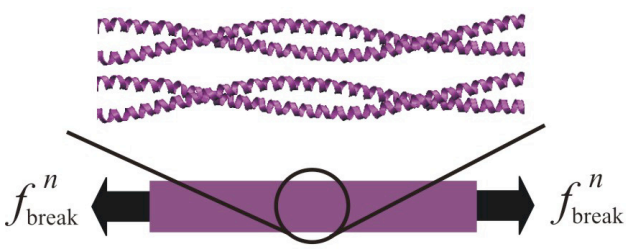

(a)

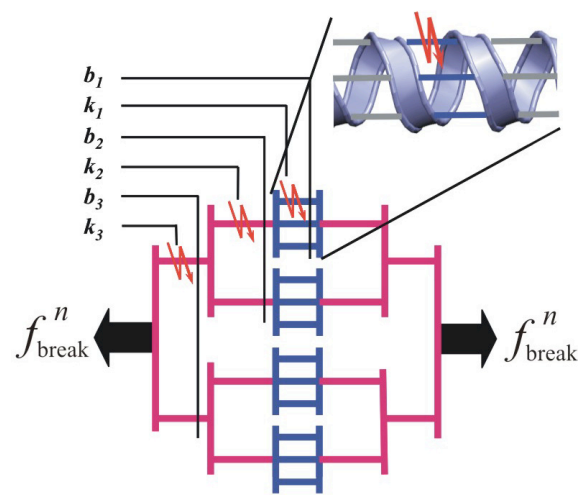

(b)

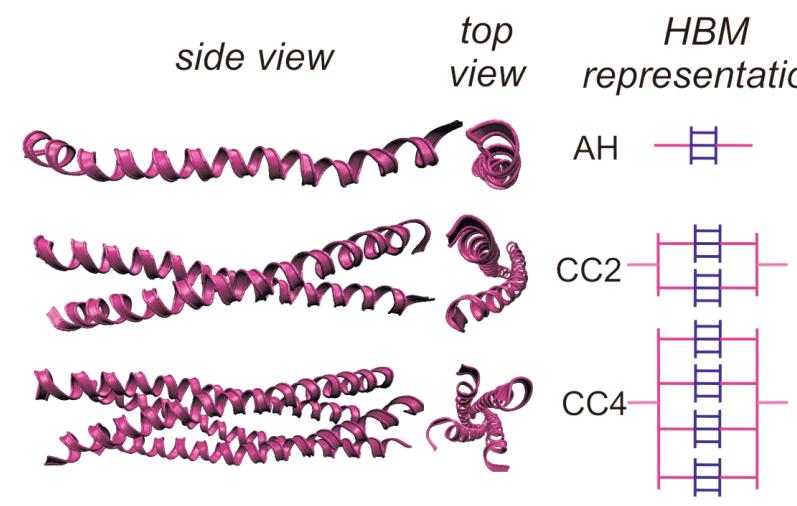

(c)

Fig. 2. Illustration of structural protein hierarchies and their representation in the Hierarchical Bell Model. Subplot (a) depicts a protein filament that consists of two CCs in parallel. Subplot (b) depicts its representation in the Hierarchical Bell Model. The inlay in the upper part of subplot $(\mathrm{b})$ shows a single $\mathrm{AH}$ structure with $\approx 3 \mathrm{H}$-bonds per convolution. Subplot (c) shows several additional AH based protein filaments and their representation in the Hierarchical Bell Model.

in Fig. 2 could in principle be used to represent other structural proteins such as beta-sheets, beta-helices or tropocollagen, since the difference between many protein structures is, similar to the case in AHs, the geometrical arrangement of H-bonds. In general, the hierarchical schematic can be applicable to any system composed of such subelements as a representation of hierarchical structure.

H-bond rupture mechanisms can be regarded as analogs to the nucleation of dislocations in ductile materials or the rupture of covalent bonds in brittle materials, representing fundamental unit deformation events [Ackbarow et al., 2007; Buehler and Ackbarow, 2007; Buehler and Gao, 2006; Buehler and Keten, 2008; Hirth and Lothe, 1982; Keten and Buehler, 2008b]. Larger-scale, effective properties of these 
hierarchical H-bond structures can not be calculated by conventional mean-field averaging approaches, not only due to an insufficient number of subelements [Ahl and Allen, 1996], but also since information may be forfeited that is crucial for the structure's behavior several scales up. Recent results provide strong evidence that the key to understand the mechanical response of $\mathrm{AH}$ based protein structures is to consider the rupture dynamics of H-bonds at mesoscale [Ackbarow et al., 2007; Dudko et al., 2006; Evans and Ritchie, 1997; Keten and Buehler, 2008b; Marszalek et al., 1999; Seifert, 2000; Sheu et al., 2003a; Sotomayor and Schulten, 2007].

\subsection{Outline of this paper}

The central question addressed in this paper is a systematic analysis of the mechanical performance of $\mathrm{AH}$ protein domains, focused on strength and robustness. We begin with a review of the Hierarchical Bell Model. In the results section, we present first an analysis of a small system of $8 \mathrm{AH}$ elements, which are arranged in different hierarchical geometries. We then extend the case study to a system with 16,384 elements. The goal of the analysis is to understand the difference in mechanical performance (strength and robustness) of different hierarchical arrangements. We conclude the paper with a broader discussion of our findings in light of de novo materials development.

\section{Theoretical Background}

\subsection{Hierarchical Bell model}

In this section we briefly review the earlier reported Hierarchical Bell Model [Buehler et al., 2008], including a discussion of its fundamental formulation approach based on the so-called "Bell model".

\subsubsection{The Bell model}

The Bell model [Bell, 1978] has been used successfully to describe the rupture mechanics of adhesion bonds, but has also been used to describe the rupture dynamics of H-bonds. The central element of Bell's model is the concept of the off rate, which describes how often a bond dissociates per unit time as a function of applied force $f$ and an energy barrier $E_{b}$ that is required to lead to bond breaking at a distance $x_{b}$ (this concept is illustrated in Fig. 3):

$$
\chi=\omega_{0} \cdot \exp \left(-\frac{\left(E_{b}-f \cdot x_{b} \cdot \cos (\theta)\right)}{k_{B} \cdot T}\right)=\omega_{0} \cdot p_{0} .
$$

In Eq. (2.1), $\omega_{0}$ is the vibration (attempt) frequency of bonds and $p_{0}$ is the probability of breaking of the bond under applied force $f$ and given the specific energy landscape parameters $x_{b}$ and $E_{b}$ as well as a given temperature $T$ (note that in Eq. (2.1) $p_{0}$ corresponds to the $\exp (.$.$) expression). This equation is based on the$ 


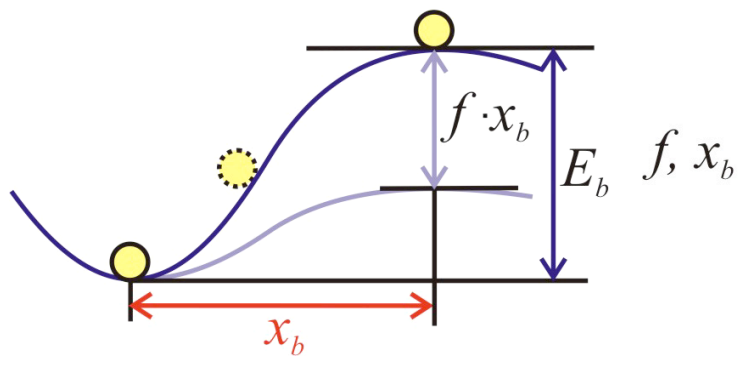

(a)

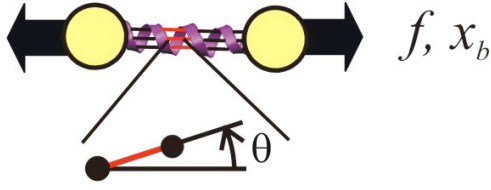

(b)

Fig. 3. Illustration of the basic concept of the Bell model. The Bell model is a statistical theory to predict the bond rupture mechanics via the bond energy landscape [Ackbarow et al., 2007; Buehler and Ackbarow, 2007]. The graph depicts the energy as a function of deformation along a deformation variable, along a particular pathway that leads to bond rupture. Here $f$ is the applied force, and $x_{b}$ is the displacement in the direction of the applied force, corresponding to the lateral displacement that is necessary to overcome the bond breaking distance of a H-bond and the associated energy barrier $E_{b}$. Given that $x_{b}^{*}$ is the distance to break a single H-bond, the distance $x_{b}^{*}=x_{b} \cos \theta$ denotes the lateral displacement at bond breaking, with the angle $\theta$ as the angle between pulling direction and orientation of the H-bond inside the molecule. This fundamental view of single bond behavior is scaled up through multiple hierarchies in the Hierarchical Bell Model, enabling us to calculate an effective energy barrier for bond breaking for a particular hierarchical structure.

concept that bond breaking is initiated by attempting to break a bond through the protein's own bond vibrations. In order to allow capturing the pulling speed dependence of the strength of $\mathrm{H}$-bonds, we link the off rate to the bond breaking speed, by multiplying the off rate by the bond breaking distance $x_{b}$, resulting in $v=\chi \cdot x_{b}$. The bond breaking speed equals the externally applied pulling speed only in the case of non-equilibrium when $v>v_{0}$, and in the case that the pulling speed is smaller than the natural bond breaking speed $v_{0}=x_{b} \cdot \omega_{0} \cdot \exp \left(E_{b} / k_{B} \cdot T\right)$ this theory is not applicable. Table 1 summarizes all parameters, variables and symbols used in this paper along with a brief description.

Existing models derived from Bell's original concept are only capable of treating conglomerates of bonds, as they only consider an effective energy barrier $\left(E_{b}\right)$ that typically corresponds to multiple individual bonds. In particular, thus far all attempts have failed to predict the strength of hierarchical arrangements of $\mathrm{H}$-bonds from fundamental principles, for example by explicitly considering the geometrical arrangements of individual H-bonds and their energetic properties. The Hierarchical Bell Model [Ackbarow and Buehler, 2009; Buehler et al., 2008] overcomes these limitations.

\subsection{Extension of the Bell model — single hierarchy systems}

We review the development of the Hierarchical Bell Model in the following sections. In order to estimate the strength and the energy landscape of a hierarchical protein structure, the original Bell model is extended to explicitly consider the structural 
Table 1. Overview over important parameters, variables and symbols used in this manuscript.

\begin{tabular}{|c|c|c|}
\hline $\begin{array}{l}\text { Mathematical } \\
\text { symbol }\end{array}$ & Unit & Description \\
\hline$E_{b}$ & $\mathrm{kcal} / \mathrm{mol}$ & Height of the energy barrier at the transition state \\
\hline$E_{b}^{0}$ & $\mathrm{kcal} / \mathrm{mol}$ & Energy barrier of a single H-bond \\
\hline$x_{b}$ & $\AA$ & Location of the energy barrier \\
\hline$f$ & $\mathrm{pN}$ & Applied force at molecule \\
\hline$\theta$ & $\circ$ (degrees) & $\begin{array}{l}\text { Angle between pulling direction and reaction coordinate of } \\
\text { breaking H-bond }\end{array}$ \\
\hline$k_{B}$ & $\mathrm{~J} / \mathrm{K}$ & Boltzmann constant \\
\hline$T$ & $\mathrm{~K}$ & Absolute temperature \\
\hline$\omega_{0}$ & $\mathrm{~s}^{-1}$ & Natural bond vibration frequency $\left(1 \times 10^{13} 1 / \mathrm{sec}\right)$ \\
\hline$\chi$ & $\mathrm{s}^{-1}$ & Off rate: bond dissociation per second \\
\hline$v$ & $\mathrm{~m} / \mathrm{s}$ & $\begin{array}{l}\text { Macroscopically: pulling speed, microscopically bond breaking } \\
\text { speed }\end{array}$ \\
\hline$v_{0}$ & $\mathrm{~m} / \mathrm{s}$ & Natural bond breaking speed, when no load is applied \\
\hline$\varepsilon$ & $\%$ & $\begin{array}{l}\text { Engineering/molecular strain: displacement of the pulled atom } \\
\text { normalized by the length of the molecule }\end{array}$ \\
\hline$b_{i}$ & - & Number of parallel elements on the hierarchy $i$ \\
\hline$k_{i}$ & - & Number of elements on hierarchy $i$ that rupture simultaneously \\
\hline$f_{v}$ & $\mathrm{pN}$ & Force contribution as a consequence of pulling speed \\
\hline$f_{h i}$ & $\mathrm{pN}$ & Force contribution as a consequence of hierarchy $i$ \\
\hline$f_{h 0}$ & $\mathrm{pN}$ & $\begin{array}{l}\text { Force contribution as a consequence of the basic hierarchy } \\
\text { (individual H-bond) }\end{array}$ \\
\hline$r$ & $\%$ & Robustness, ranging from $0 \%$ to $100 \%$ \\
\hline$N$ & - & Number of hierarchies in a structure \\
\hline$\eta$ & - & Number of hierarchical degrees of freedom (HDOFs) \\
\hline
\end{tabular}

hierarchies of the protein structure with the only input parameters being the energy of an H-bond and the rupture distance. The H-bonds thereby form a hierarchical structure, reaching from individual H-bonds at the lowest, atomistic level to a collection of H-bonds at the next higher, molecular protein scale.

In the Hierarchical Bell Model, the lowest hierarchy is represented by an individual H-bond (hierarchy level 0) with an $E_{b}^{0}$ and $x_{b}$, and the higher hierarchy consists of parallel H-bonds (hierarchy 1 ). Here $b_{1}$ describes the number of bonds in a structure that are in parallel, where $k_{1}$ bonds out of these $b_{1}$ bonds break simultaneously during failure. The existence of multiple bonds changes the probability for failure initiation, where a larger number of bonds provide an increased number of degrees of freedom for vibrational modes, thus effectively reducing the likelihood for one of the bonds to fail. In particular, as with each force vibrational attempt $k_{1}$ out of $b_{1} \mathrm{H}$-bonds are attempted, this results in

$$
{ }_{b_{1}} C_{k_{1}}=\left(\begin{array}{c}
b_{1} \\
k_{1}
\end{array}\right)
$$

(binomial coefficient) possible attempts. The probability that one of these combinations constitutes a particular rupture event is one divided by ${ }_{b_{1}} C_{k_{1}}$, since ${ }_{b_{1}} C_{k_{1}}$ more attempts are necessary to break the system. Also, if $k_{1}$ bonds break simultaneously in the fundamental rupture event, the total energy barrier increases by a 
factor $k_{1}$, to $k_{1} \cdot E_{B}^{0}$. This leads to the following expression for the off rate:

$$
\chi_{H}=\omega_{0} \cdot\left(\begin{array}{l}
b_{1} \\
k_{1}
\end{array}\right)^{-1} \cdot \exp \left(-\frac{\left(k_{1} \cdot E_{B}^{0}-f \cdot x_{b} \cdot \cos (\theta)\right)}{k_{B} \cdot T}\right) .
$$

It is noted that Eq. (2.3) can be expressed as $\chi=\omega_{0} \cdot\left(\begin{array}{l}b_{1} \\ k_{1}\end{array}\right)^{-1} p_{0}$. We rewrite Eq. (2.3) so that the binomial coefficient appears in the exponential, which enables us to compare with Eq. (2.1):

$$
\chi_{H}=\omega_{0} \cdot \exp \left(-\frac{\left(k_{1} \cdot E_{b}^{0}+k_{B} \cdot T \cdot \ln \left(\begin{array}{l}
b_{1} \\
k_{1}
\end{array}\right)-f \cdot x_{b} \cdot \cos (\theta)\right)}{k_{B} \cdot T}\right) .
$$

The parameter $E_{b}$ in Eq. (2.1) represents the "effective" energy barrier, and by comparison with Eq. (2.4) can be split it up as follows:

$$
E_{b}=k_{1} \cdot E_{b}^{0}+k_{B} \cdot T \cdot \ln \left(\begin{array}{c}
b_{1} \\
k_{1}
\end{array}\right)
$$

where $E_{b}^{0}$ is the energy of a single bond and the term $k_{B} \cdot T \cdot \ln \left(\begin{array}{c}b_{1} \\ k_{1}\end{array}\right)$ is the contribution to the "effective" energy barrier due to the hierarchical structure, resulting from the increased level of uncertainty. The underlying physical concept is comparable to entropic effects, where a larger number of degrees of freedom (due to the larger number of available bonds at this hierarchical level) makes it more difficult for the system to fail as the vibrational energy can be distributed into a larger number of modes. The hierarchical structure essentially provides additional complexity to the energy landscape of the system, increasing the total number of possible energy states for a given loading condition. This behavior of systems in dependence of the number of degrees of freedom is similar to the observation that the compressibility of ideal gases increase with increasing temperature or entropy, as kinetic energy can be stored in more accessible modes.

By substituting $v=\chi \cdot x_{b}$ and solving for $f$, the resulting protein domain rupture force is given by

$$
f\left(v, b_{1}, k_{1} ; E_{b}^{0}, x_{b}, \theta\right)=\frac{k_{B} \cdot T}{x_{b} \cdot \cos \theta} \ln \left(\frac{v}{x_{b} \cdot \omega_{0}}\right)+\frac{k_{B} \cdot T}{x_{b} \cdot \cos \theta} \cdot \ln \left(\begin{array}{c}
b_{1} \\
k_{1}
\end{array}\right)+\frac{k_{1} \cdot E_{b}^{0}}{x_{b} \cdot \cos \theta} .
$$

It is noted that $f=f_{v}+f_{h 1}+f_{h 0}$, where the parameters $f_{v}, f_{h 1}$ and $f_{h 0}$ are the contributions to the force as a consequence of the pulling speed, the first hierarchy level (number of parallel bonds, $b_{1}$ ), and the basic hierarchy (strength of bonds, $E_{b}^{0}$ and $x_{b}$ ). This expression quantifies how the hierarchical design influences the rupture strength, for one level of hierarchy. 


\subsection{Multiple hierarchy systems}

As shown in the previous section, the introduction of the first hierarchy led to the introduction of the term ${ }_{b_{1}} C_{k_{1}}$. This means that the existence of multiple elements increases the number of possibilities in that system failure $\left(={ }_{b_{1}} C_{k_{1}}\right)$ can be attempted at the attempt frequency $\omega_{0}$.

To generalize this concept, we define $\eta$ as the number of hierarchical degrees of freedom (HDOFs), measuring how the existence of hierarchical structures influences the number of available vibrational modes. Since for the initiation of failure of the system $\eta$ times more attempts are necessary, a strengthening of the system with increasing number of HDOFs is observed. Thus the parameter $\eta$ can be linked to the level of uncertainty of a system. This results in the following expression for the off rate: $\chi_{H}=\omega_{0} \cdot \eta^{-1} \cdot p_{0}$, where $p_{0}$ as the reference probability for bond failure, and $\omega_{0}$ is the vibration or attempt frequency and $\eta$ the number of HDOFs (for the single hierarchy system), $\eta={ }_{b_{1}} C_{k_{1}}$.

How can the number of HDOFs $\eta$ be calculated for a system consisting of two, three or in general terms $N$ levels of hierarchy? For the extension to higher levels of hierarchy we use an analogy from structural dynamics, by assuming coupling between the degrees of freedom at a common structural level [Chopra, 2007]. Consequently, for two levels of hierarchy, that is, at system consisting of $b_{2}$ elements each with $b_{1}$ subunits (here we show system behavior only for the case of $k_{i}=1$ for $i>1$, corresponding to only one bond in the first hierarchy breaks before the failure of the filament), the total number of HDOFs can be considered as follows:

$$
\eta=b_{2} \cdot b_{1}^{b_{2}} .
$$

This can be linked to the coupling of modal shapes of vibration in structural dynamics. The exponent $b_{2}$ describes in this equation the $b_{2}$ systems at the largest hierarchy level (each with $b_{1}$ independent HDOFs), and the prefactor $b_{2}$ describes the number of HDOFs of the largest hierarchy level that consists of $b_{2}$ elements. Notably, by not considering the exponent the equation would describe a system where $b_{2}$ elements (each with $b_{1}$ HDOFs) are coupled at one single level of hierarchy, thus not considering any hierarchical effect.

We extend the analogy to structural mechanics a little further for additional clarification. A HDOF does not represent a discrete point or value (that is, a single $\mathrm{H}$-bond), but rather the effect of the hierarchical unit on the entire system. A system description is obtained by the superposition of all HDOFs, similar to the superposition of modal shapes and corresponding eigenvalues in structural dynamics [Chopra, 2007]. Thus HDOFs should not be thought of in terms of discrete physical degrees of freedom corresponding in a one-to-one relationship with the hierarchical elements. Similar to structural dynamics, knowing the possible modal shapes of a structure does not directly indicate the displacements of specific points (commonly associated with degrees of freedom). In this sense, knowing all the hierarchical degrees of freedom does not completely describe the state of the system - additional data is 
required. In modal decomposition, such information is derived from system information (e.g. member stiffnesses, applied forces, etc.). We can likewise define the HDOFs simply by knowing the geometry of the hierarchies - independent of the structural or other system information - but the state of the system is not fully described. In terms of coupling the hierarchies, we can consider the total energy of the system. In this case, each HDOF represents the bond energy (as opposed to a modal displacement), and the combination of HDOFs describes the possible energy landscape of the system. Considering all hierarchical levels, the system energy is finite and increasing or decreasing the HDOFs of one of the contributions will affect the others.

This can be clarified by considering a simple system with coupled degrees of freedom, such as a system of ideal gas at constant temperature. The kinetic energy of each atom can be considered as a degree of freedom, but each is coupled at the system level to maintain a constant temperature. Likewise, the contribution of each HDOF has an effect on the system's energy landscape and dependent on the state of the total number of system HDOFs, thus the system is inherently coupled.

Alternatively, coupling can be explained at a larger scale returning to a structural mechanics analogy, considering a model with two simple structures with active viscous dampers. For example, consider two isolated moment frames under seismic loading, free to vibrate independently. The modal shapes are not coupled. Introducing active viscous dampers between the two systems dynamically changes the modal shapes - i.e. the modal degrees of freedom of each moment frame is coupled to the other [Chopra, 2007]. Similarly, the contribution of a specific HDOF will have an effect on all the HDOFs of the system. In this case, it is not intended to imply that hierarchical elements impose a kind of energetic damping effect, but is merely compared for illustrative purposes.

Additionally, system information or assumptions can result in variations of the number of HDOFs (due to system specific conditions such as stiff linkers, fixities, etc., in determining the depth of hierarchical coupling). Moving to a system with three levels of hierarchy; in which all levels are coupled (maximum hierarchical depth of coupling), results in the following expression:

$$
\eta=b_{3}\left(b_{2} \cdot b_{1}^{b_{2}}\right)^{b_{3}}=b_{3} \cdot b_{2}^{b_{3}} \cdot b_{1}^{b_{2} b_{3}} .
$$

This means that the elements on each hierarchical scale are coupled with each other. This seems, however, to be an unrealistic assumption for systems with a very high number of hierarchies, as information between each element on the lowest hierarchical scale are transported at a finite speed during rupture. Thus we adapt a simplified assumption that elements are coupled only to their next-nearest neighbors (in the sense of a nearest neighbor cutoff as it is used in interatomic potentials, for instance), thus leading to:

$$
\eta=b_{3} \cdot b_{2}^{b_{3}} \cdot b_{1}^{b_{2}}
$$


This idea can be extended and generalized for a system with $N$ levels of hierarchy as follows (including cases where $k_{1} \neq 1$, corresponding to $k_{1} \mathrm{H}$-bonds in the first hierarchy break simultaneously, initiating system failure):

$$
\eta=b_{n} \cdot \prod_{i=2}^{N-1}\left(b_{i}^{b_{i+1}}\right) \cdot\left(\begin{array}{c}
b_{1} \\
k_{1}
\end{array}\right)^{b_{2}} .
$$

For this model we assume that for the second and higher level hierarchies $k_{i}=1$ for $i>1$ and that the rupture of one element leads to catastrophic failure of the whole system, representing a highly cooperative process.

The above mentioned relation $\left(\chi_{H}=\omega_{0} \cdot \eta^{-1} \cdot p_{0}\right)$ can now be implemented together with equation (2.10) into equation (2.1), resulting in:

$$
\begin{aligned}
\chi= & \eta^{-1} \cdot \omega_{0} \cdot p_{0}=\left(b_{n} \cdot \prod_{i=2}^{N-1}\left(b_{i}^{b_{i+1}}\right) \cdot\left(\begin{array}{c}
b_{1} \\
k_{1}
\end{array}\right)^{b_{2}}\right)^{-1} \\
& \cdot \omega_{0} \cdot \exp \left(-\frac{\left(k_{1} \cdot E_{b}^{0}-f^{h N} \cdot x_{b} \cdot \cos (\theta)\right)}{k_{B} \cdot T}\right)
\end{aligned}
$$

By considering $v=\chi \cdot x_{b}$, this can be solved for $f^{h N}$ and the energy barrier $E_{b}^{h N}$ of a system consisting of $N$ hierarchies as follows:

$$
\begin{aligned}
f^{h N}= & \frac{k_{B} \cdot T}{x_{b} \cdot \cos \theta} \cdot\left[\ln \left(\frac{v}{x_{b} \cdot \omega_{0}}\right)+\ln \left(b_{n}\right)+\sum_{i=2}^{N-1} b_{i+1} \cdot \ln \left(b_{i}\right)+b_{2} \cdot \ln \left(\begin{array}{l}
b_{1} \\
k_{1}
\end{array}\right)\right] \\
& +\frac{k_{1} \cdot E_{b}^{0}}{x_{b} \cdot \cos \theta}=f_{v}+\sum_{i=1}^{N} f_{h i}+f_{h 0}
\end{aligned}
$$

and the effective energy barrier for the hierarchical system is given by

$$
E_{b}^{h N}=k_{1} \cdot E_{b}^{0}+k_{B} \cdot T \cdot\left[\ln \left(b_{n}\right)+\sum_{i=2}^{N-1} b_{i+1} \cdot \ln \left(b_{i}\right)+b_{2} \cdot \ln \left(\begin{array}{c}
b_{1} \\
k_{1}
\end{array}\right)\right]=E_{b} .
$$

These equations enable us to predict the unfolding force at any pulling speed, once the structural geometry and the energy of a single $\mathrm{H}$-bond, $E_{b}^{0}$, is known. Recall that the parameter $b_{i}$ denotes the number of parallel elements at level hierarchy $i$, and $k_{i}$ specifies the number of elements on hierarchy $i$ that rupture simultaneously (we assume that only $k_{1}$ can deviate from one, where other cases will be treated in the future studies). Equation (2.13) provides an explicit expression for the effective energy barrier as a function of the hierarchical protein structure and the energy barrier of an individual H-bond, $E_{b}^{0}$. Further, these equations enable us to predict the rupture force $f^{h N}$ at any pulling speed, once the structural geometry and the energy landscape of a single H-bond is known. 
Interestingly, by rewriting Eq. (2.12) we receive the following energy balance:

$$
E_{\text {ext }}=f^{h N} \cdot x_{b} \cdot \cos \theta=k_{B} \cdot T \cdot\left[\ln \left(\frac{v}{x_{b} \cdot \omega_{0}}\right)+\ln (\eta)\right]+k_{1} \cdot E_{b}^{0}=E_{\text {int }} .
$$

Equation (2.14) shows that the externally applied work (force moving along the reaction coordinate) equals to the change of internal energy. The energy is used inside the system to (1) rupture $k_{1} \mathrm{H}$-bonds and (2) to overcome the level of uncertainty, that is, the entropic effects measured by $\eta$.

We note that the Hierarchical Bell Model utilized here only considers H-bonds as structural elements in the definition of AH-based protein structures, representing a limitation of this description. Thereby it does not consider hydrophobic effects and other chemical interactions between molecules, which may play a role in defining the strength properties. This is a limitation of the model; however, there is currently no method to explicitly include these effects in the model and thus this task is left to future work.

We expect that the effect of intermolecular adhesion is limited with respect to the prediction of the initial strength values. This is based on our observation that in $\mathrm{AH}-$ based $\mathrm{CCs}$ protein structures, failure initiates first in the individual AHs (thus defining its strength properties) and is later followed by uncoiling of the overall coiled-coil structure [Ackbarow and Buehler, 2007]. Further, the direction of Hbonds is mainly along the pulling direction, whereas the force direction of adhesion effects in CCs is perpendicular to the protein axis, holding individual AHs together. This suggests that the approach taken here by focusing solely on H-bonds is a good approximation for the strength properties of $\mathrm{AH}$ based protein domains. It is noted that the Hierarchical Bell Model as presented above has been validated against a series of MD simulations, as reported in earlier work [Ackbarow and Buehler, 2009; Buehler et al., 2008].

\subsection{Definition of robustness in biological protein materials}

We calculate robustness as the ratio of strength of a defected system and an intact system, by following Kitano's definition of robustness as fault insensitivity [Kitano, 2002]. For the calculations reported below, the intact system is defined as a system in which all AHs contribute to strength (see Eq. (2.12) for the definition of $f_{h i}$, here no pulling speed effects are considered as they are the same in both systems), whereas in the defected system all except one AHs (fault on second hierarchy, variation of $b_{2}\left[=\right.$ number of AHs in a bundle] resulting in $b_{2}^{*}$ ) contribute to the strength. This results in the following expression:

$$
\begin{aligned}
r\left(b_{i}\right) & =\frac{f\left(b_{i}, k_{i}, b_{2}^{*}=b_{2}-1\right)}{f\left(b_{i}, k_{i}\right)} \\
& =\frac{f_{h 0}+f_{h 1}\left(k_{1}=3, b_{1}=3\right)+f_{h 2}\left(k_{2}=1, b_{2}^{*}=b_{2}-1\right)+\sum_{i=3}^{N} f_{h i}\left(k_{i}=1, b_{i}\right)}{f_{h 0}+f_{h 1}\left(k_{1}=3, b_{1}=3\right)+\sum_{i=2}^{N} f_{h i}\left(k_{i}=1, b_{i}\right)}
\end{aligned}
$$


Robustness converges towards complete fault tolerance when $b_{i} \rightarrow \infty$ (as shown in earlier studies [Ackbarow and Buehler, 2009; Buehler et al., 2008]). We note that other robustness perspectives in biological materials include adaptation to environmental changes, as well as graceful degradation [Kitano, 2002]. This definition of robustness is related to a structure's ability to tolerate structural defects; it is therefore related to the material properties of toughness. Tough materials show high robustness against catastrophic failure despite the presence of a defect. Notably, the fault could be set on other hierarchical levels (e.g. at the level of H-Bonds or coiled-coil proteins), which would lead to qualitatively the same behavior.

\section{Results}

The Hierarchical Bell Model reviewed above is now used in a series of theoretical analyses of case studies, addressing the question, how are AH based protein domains capable of unifying strength and robustness, and how does their mechanical performance depend on the specific structural geometry? Here we limit our analysis to the theoretical study based on the Hierarchical Bell Model, as the direct atomistic simulation of the systems considered here is not possible due to computational limitations (albeit the parameters used in the Hierarchical Bell Model are directly determined for single AHs from MD simulations).

For the following analysis we use the $E_{b}^{0}$ from the single $\mathrm{AH}\left(E_{b}^{0}=4.21 \mathrm{kcal} / \mathrm{mol}\right.$ and $x_{b}=1.21 \AA$ ) as extracted from full atomistic molecular dynamics simulations of AH structures at $T=300 \mathrm{~K}$ [Ackbarow et al., 2007] (this corresponds to the unit mechanism of failure). It is noted that these values are also close to results of experimental and theoretical investigations of the rupture mechanism of $\mathrm{AH}$ protein domains [Ackbarow et al., 2007; Sheu et al., 2003b]. Earlier full atomistic studies in explicit water solvent by the same authors have shown that the fundamental fracture mechanism of a single AH element is the simultaneous rupture of approximately 3 H-bonds [Ackbarow et al., 2007]. This mode represents the basic unit mechanism of rupture in vivo, at pulling rates below $0.1 \mathrm{~m} / \mathrm{sec}$ [Ackbarow et al., 2007]. This mechanism is considered in the case studies reported here, represented by $k_{1}=3$, thereby providing a realistic description of the behavior closer to experimentally accessible pulling rates (it was shown in an earlier analysis [Ackbarow et al., 2007] that this mode of deformation, $k_{1}=3$, dominates at low deformation rates).

To present the results, we use the following nomenclature $\left\{b_{N}, b_{N-1}, \ldots, b_{2}, b_{1}\right\}$ to uniquely describe the various hierarchical structures. The values of $b_{i}$ in this expression thereby define the number of elements that are found in parallel with each other at a particular hierarchical level, from the largest to the smallest elements. Since for all cases considered here $b_{1}=3$ (since we consider AHs as basic constituents), we do not include the last term and simply write $\left\{b_{N}\right.$, $\left.b_{N-1}, \ldots, b_{2}\right\}$. 


\subsection{Low-level hierarchical systems}

We begin our analysis by studying an arrangement of eight single AHs in different hierarchical structures (that is, $b_{N} \cdot b_{N-1} \cdot b_{2}=8$ ). We ask the question, how can one arrange eight AHs to obtain different levels of robustness and strength? Figure 4(a) displays how these eight AHs are arranged into four possible hierarchical systems. The systems consist of two, three and four hierarchies. Figure 4(b) shows the performance of these four structures in the strength-robustness domain (we normalize the force by the number of AHs in order to allow the comparison between systems with different number of AHs, as they will be introduced later). Notably, the differences in robustness and strength calculated with the Hierarchical Bell Model are not achieved through additional use of materials, but purely through different hierarchical arrangements of the constitutive elements. The overall behavior of the different structural arrangements lay on a curve that resembles the overall curvature of the curve shown in Fig. 1(b) (albeit no quantitative comparison is made here), leading to an inverse banana-curve.

In the robustness-strength map, the "best" material behavior is the one in which high robustness is achieved at large strength (for this case, both parameters need to be optimized; where the best performance is defined as the one that maximizes the product of strength and robustness). It can be seen that system 2 $\{2,4\}$ is the best performer. Notably, it is not the system with the highest hierarchical level (system 4), nor the system with the highest level of redundancies (system 1). In other words, system 2 features the best combination of redundancies at different hierarchical levels, and features superior mechanical performance without additional material use. The physical representation of system $2\{2,4\}$ is a bundle of two CC4s (CC4s are coiled protein domains composed of four AHs). System $3\{4,2\}$ is the configuration with highest strength. The physical representation of this system is a four-fold bundle of CC2s (coiled-coil protein domains). The highest robustness is achieved for a lowest hierarchy system with 8 parallel AHs (system $1,\{8\}$ ).

Figure 4(c) shows the same analysis for $16 \mathrm{AH}$ elements. The behavior observed for this case is very similar to the previous case, where the various structures also lie on an inverse banana-curve. For this case, the best performer is $\{4,4\}$. The physical representation of $\{4,4\}$ is a bundle of four CC4s. The system $\{8,2\}$ is the configuration with highest strength. The physical representation of this system is an eight-fold bundle of CC2s. The highest robustness is achieved for a lowest hierarchy system with 16 parallel AHs, system $\{16\}$.

Notably, for both cases, the best performer for both high robustness and high strength are structures of the $\{x, 4\}$ family, where $x=2$ for the smaller and $x=4$ for the larger system. Furthermore, for both cases the systems with highest strength are CC2 systems of the $\{x, 2\}$ family, where $x=4$ for the smaller and $x=8$ for the larger system. 


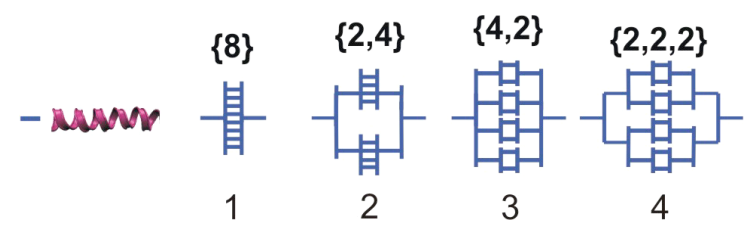

(a)

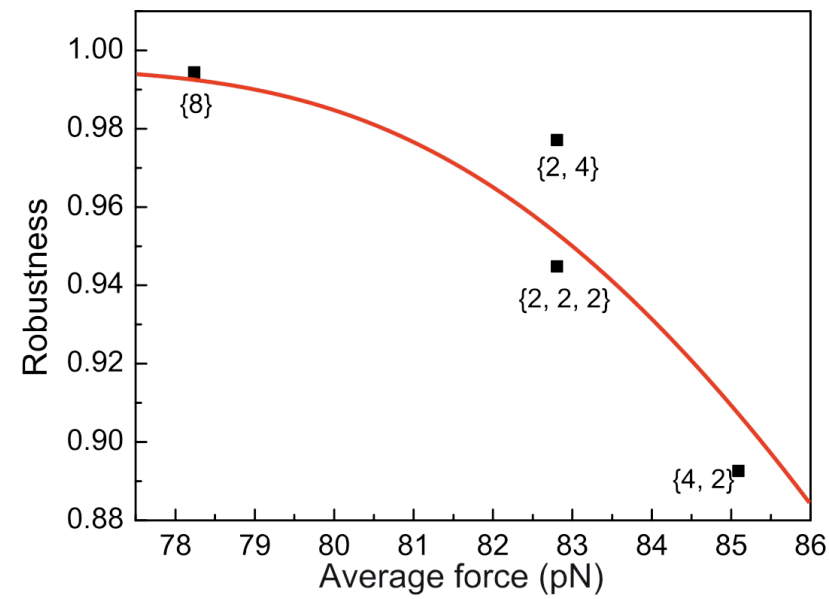

(b)

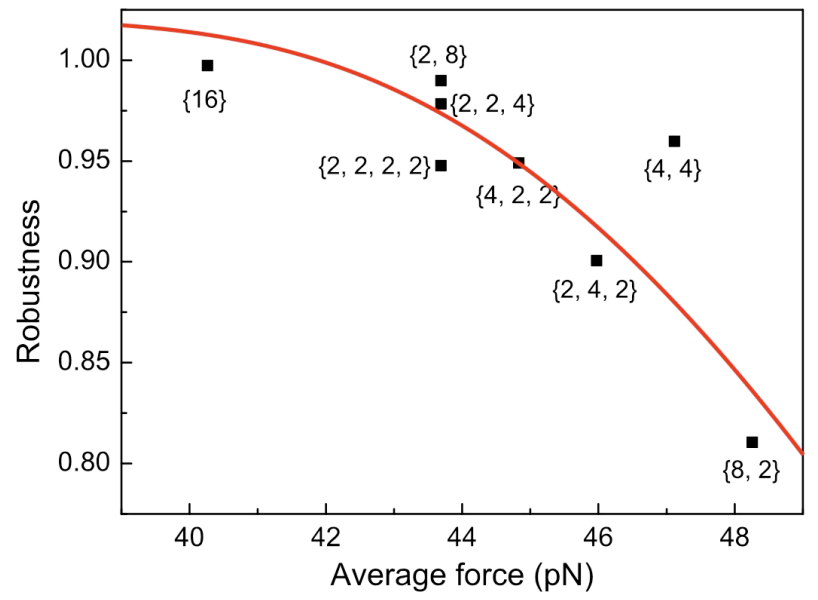

(c)

Fig. 4. The strength-robustness relation for alpha-helical protein filament with few (8 and 16) AH subelements. Subplot (a) shows the geometries of the hierarchical structures considered (for 8 elements). Subplot (b) depicts results for 8 subelements in the protein filament. The definition of subelements and their arrangement are shown in subplot (a). Subplot (c) depicts results for 16 subelements in the protein filament. 


\subsection{High-level hierarchical systems}

If the number of elements increases significantly, different domains of mechanical performance can be observed in the strength-robustness plot. Figure 5(a) shows results for $128 \mathrm{AH}$ elements, and Fig. 5(b) shows results for 16,384 AH elements.

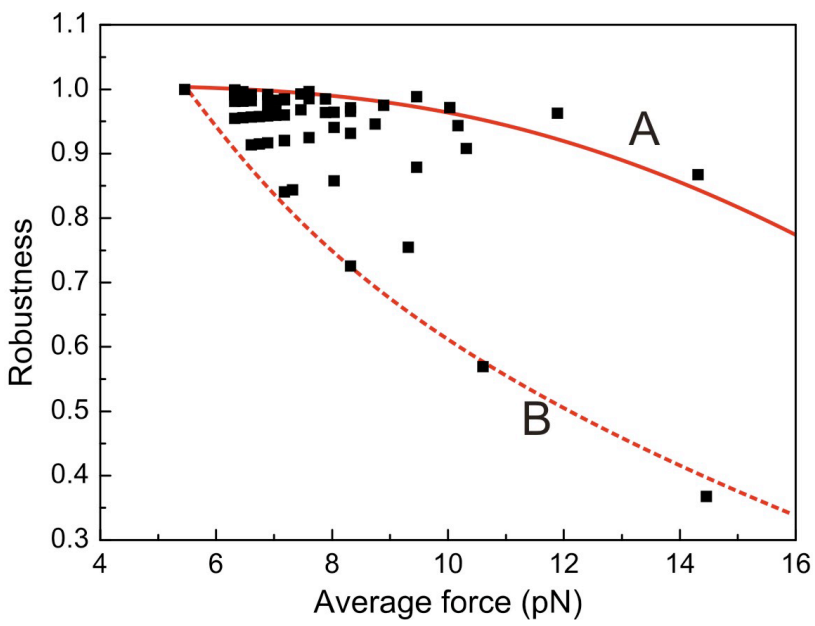

(a)

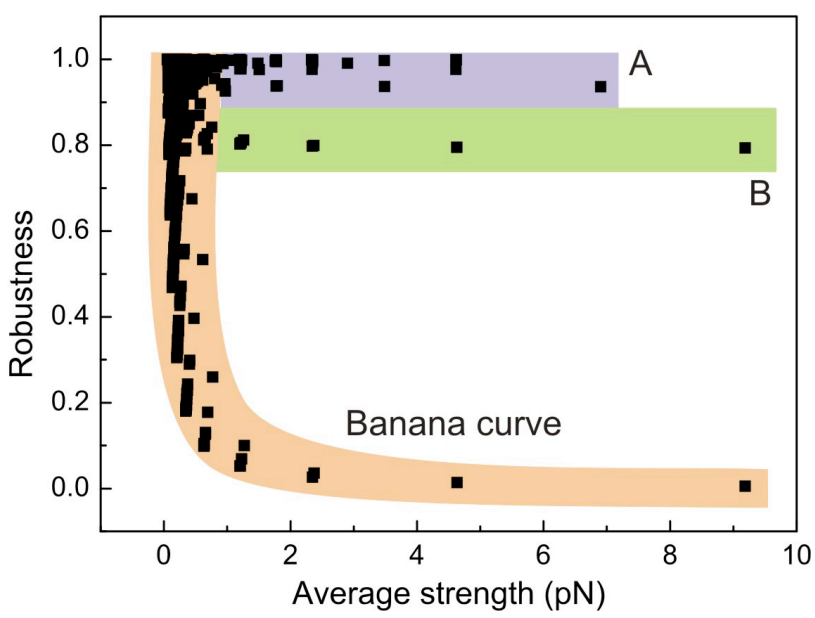

(b)

Fig. 5. The strength-robustness relation for alpha-helical protein filament with a larger number (128 and 16,384) AH subelements. Subplot (a) depicts results for 128 subelements in the protein filament. The results show in addition the curve indicated by "A", there occurs development of a secondary "cluster" "B", following a curve similar to the banana curve. Subplot (b) depicts results for 16,384 subelements in the protein filament. The percentage values in subplot (b) denote how many configurations lie in the respective domain (as indicated in the plot, for regions A and B as well as the banana curve domain). 
Note that as before, the constraint for the system size is given by $b_{N} \cdot b_{N-1} \cdot b_{2}=128$ and $b_{N} \cdot b_{N-1} \cdot b_{2}=16,384$, respectively. Unlike in the results shown in Section 3.1 for relatively few constitutive elements, for 128 elements we observe the emergence of two distinct domains in the mechanical performance (as indicated with the continuous and dashed line in Fig. 5(a)). Points cluster, as before, along an inverse-banana curve. However, a second curve can be recognized, which resembles the banana curve behavior.

In the robustness-strength map, the "best" material behavior with high robustness at large strength is $\{32,4\}$. As for the previous cases it is not the system with the highest hierarchical level, nor the system with the highest level of redundancies. The strongest system is $\{64,2\}$, and the most robust system is given by $\{128\}$. The physical representation of $\{32,4\}$ is a bundle of $32 \mathrm{CC} 4 \mathrm{~s}$, where the physical representation of $\{64,2\}$ is an 64 -fold bundle of CC2s. As before, the differences in robustness and strength are not achieved through additional use of materials, but purely through different hierarchical arrangements of the constitutive $\mathrm{AH}$ elements.

The trend of emergence of formation of two distinct clusters of mechanical performance as seen in the case of 128 elements is even more clear in the largest system considered here, with 16,384 elements (see Fig. 5(b)). Here there is a clearly visible banana-curve type behavior where strength and robustness are exclusive properties, as $r \sim 1 / f$. In addition, another cluster of data points emerges that contains points that combine high robustness at high levels of strength. In this cluster, the best performer is the $\{4096,4\}$ system. The strongest system is $\{8192,2\}$, and the most robust system is given by $\{16384\}$. The physical representation of $\{4096,4\}$ is a bundle of 4,096 CC4s, where the physical representation of $\{8192,2\}$ is an 8,192 -fold bundle of CC2s.

An analysis of the distribution of the number of systems in the different clusters provides some interesting insight (see Fig. 6). Most random combinations of elements $(>98 \%)$ lead to either high strength or high robustness, reflecting the so-called banana-curve performance in which strength and robustness are mutually exclusive properties. In contrast, for few specific types of combinations of the

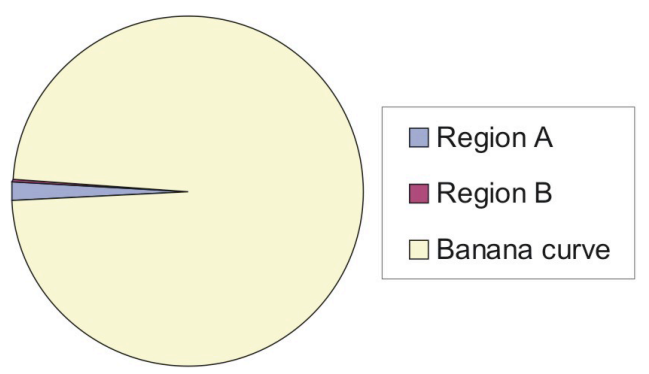

Fig. 6. Structural analysis of mechanical characteristics. The analysis shows that most data points (>98\%) in Fig. 5(b) fall onto the banana-curve. Only less than $2 \%$ of all structures lead to high strength and high robustness. 
elements in hierarchies $(<2 \%)$ it is possible to maintain high strength at high robustness levels. This behavior is reminiscent of naturally observed material performance in biological materials, where the existence of particular hierarchical structures enables to fundamentally change the material performance.

The results suggest that biological materials may have developed under evolutionary pressure to yield materials with multiple objectives, such as high strength and high robustness, a trait that can be achieved by utilization of hierarchical structures. It also shows that the particular arrangement of constituents at nanoscale matters, and that a particular design is required in order to observe the unique mechanical traits founds in biological materials. Further, this observation explains the relatively poor mechanical performance of engineered materials. These materials typically contain random arrangements of nanostructural elements (due to a lack of hierarchical structures, e.g. the microstructure of grains in polycrystalline metals are typically randomly arranged). Since most random arrangements $(>98 \%)$ lead to the banana-curve type behavior, we would expect such performance for materials with a lack of appropriate hierarchical structural designs.

Notably, as before the best performer for both high robustness and high strength are structures of the $\{x, 4\}$ family, where $x=32$ for the smaller and $x=4096$ for the larger system. Furthermore, for both cases the systems with highest strength are CC2 systems of the $\{x, 2\}$ family, where $x=64$ for the smaller and $x=8192$ for the larger system. The mechanical performance of other cases is summarized in Table 2, where it can be confirmed that this appears to be a general rule applicable to a variety of system sizes.

Interestingly, no matter how many elements are present, systems with three levels of hierarchy are preferred for both highest strength per $\mathrm{AH}$ and for best overall performance with high strength and high robustness, despite the fact that much

Table 2. Analysis of mechanical performance for different number of elements, picking out the system geometry that maximizes the product of strength and robustness and the one that maximizes strength only. We find that all systems that maximize strength and robustness are all based on the $\mathrm{CC} 4$ structure. All systems with maximum strength are based on the CC2 structure.

\begin{tabular}{ccc}
\hline \# Elements & $\begin{array}{c}\text { Max (strength.robustness) case } \\
\left\{\mathrm{b}_{n}, \mathrm{~b}_{n-1} \ldots \mathrm{b}_{2}\right\}\end{array}$ & $\begin{array}{c}\text { Max } \\
\left\{\mathrm{b}_{n}, \mathrm{~b}_{n-1} \ldots \mathrm{b}_{2}\right\}\end{array}$ \\
\hline $\mathbf{1 6 3 8 4}$ & $\{4096,4\}$ & $\{8192,2\}$ \\
$\mathbf{8 1 9 2}$ & $\{2048,4\}$ & $\{4096,2\}$ \\
$\mathbf{4 0 9 6}$ & $\{1024,4\}$ & $\{2048,2\}$ \\
$\mathbf{2 0 4 8}$ & $\{512,4\}$ & $\{1024,2\}$ \\
$\mathbf{1 0 2 4}$ & $\{256,4\}$ & $\{512,2\}$ \\
$\mathbf{5 1 2}$ & $\{128,4\}$ & $\{256,2\}$ \\
$\mathbf{2 5 8}$ & $\{64,4\}$ & $\{128,2\}$ \\
$\mathbf{1 2 8}$ & $\{32,4\}$ & $\{64,2\}$ \\
$\mathbf{6 4}$ & $\{16,4\}$ & $\{32,2\}$ \\
$\mathbf{3 2}$ & $\{8,4\}$ & $\{16,2\}$ \\
$\mathbf{1 6}$ & $\{4,4\}$ & $\{8,2\}$ \\
$\mathbf{8}$ & $\{2,4\}$ & $\{4,2\}$ \\
\hline
\end{tabular}


higher levels of hierarchy could be constructed based on the number of elements. This results from a logarithmic increase in force with an increasing number of AHs (increased material use). Remarkably, in Nature only a handful of hierarchies, as they were shown here, are found on a particular level (e.g. IF filaments, or certain levels of hierarchies in bone). By increasing the length and timescale other geometrical arrangements are applied.

To further illustrate this point, Fig. 7 shows additional analysis of the largest system with 16,384 elements. This figure depicts the relation between hierarchy number and strength (Fig. 7(a)) as well as between hierarchy number and robustness (Fig. 7(b)). The results in Fig. 7(a) show that systems with three levels of hierarchies lead to the highest strength. For robustness, the data shown in Fig. 7(b) indicate that as the number of hierarchical levels is altered, the accessible values for robustness changes as well. For few and high levels of hierarchies, robustness values tend to be high (note that the best performer $\{4096,4\}$ is indicated in the plot). For intermediate levels of hierarchies (between 3 and 8, approximately) the accessible robustness values range widely from almost 0 to 1 .

\section{Discussion and Conclusions}

We have shown here that with different structural arrangements, different combinations of strength and robustness can be achieved. This finding is the most important result of the case studies put forth in this article: It illustrates that the conflict between strength and robustness can be resolved by introducing hierarchies as an additional design variable.

Other important contributions of this paper are:

- The use of hierarchies represents an additional design variable that enables us to overcome the disparity between strength and robustness (see, e.g. Figs. 4 and 5). At the same material use, hierarchical structures are capable of combing high strength and high robustness, referred to as the inverse behavior. These results suggest that hierarchies are the key to enable the type of material performance as seen in biological structures (see, Fig. 1(a)).

- The particular type of hierarchical structure matters. For a large number of constitutive elements $(16,384)$ most "random" hierarchical structures fall onto the so-called banana curve on which strength and robustness remain exclusive. Only less than $2 \%$ of all structures provide the inverse behavior. This behavior has been illustrated in Figs. 5(b) and 6.

- This distribution of performance characteristica for a large number of elements may explain why most engineered materials show a poor performance of strength and robustness. Most randomly picked arrangements fall on the banana curve (see also Figs. 5(b) and 6). Up until now, the hierarchical nanostructural geometries have not yet been utilized for most engineering applications (e.g. materials design). Our results show that very specific geometries are required to achieve 


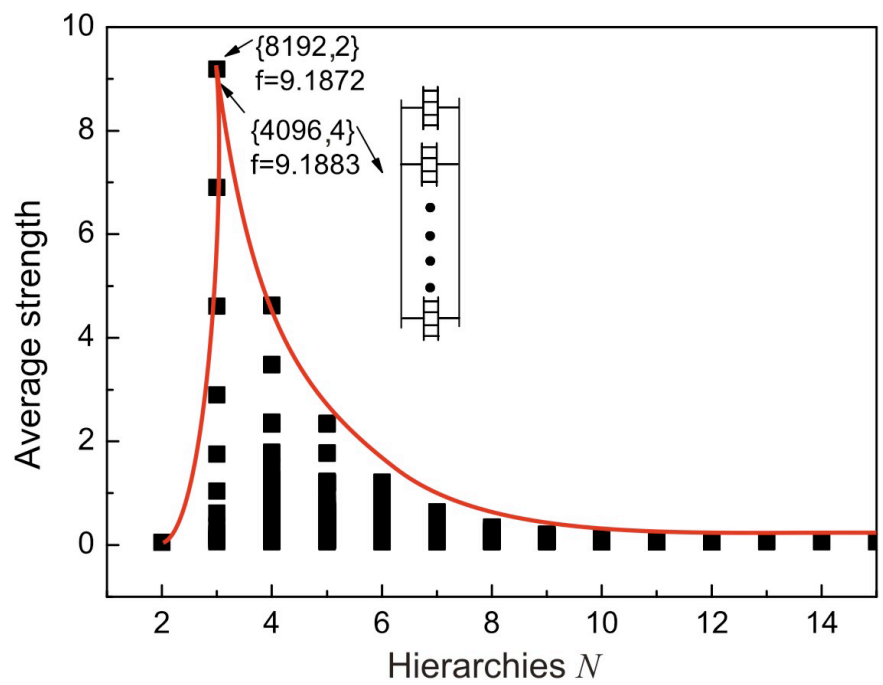

(a)

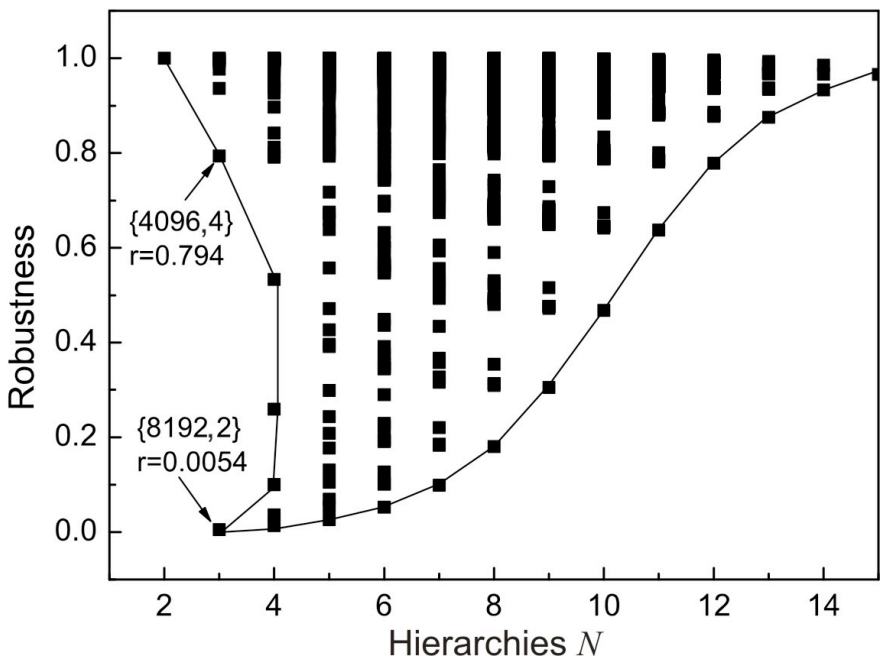

(b)

Fig. 7. Relation of hierarchy number $N$ and average strength (subplot (a)) as well as between hierarchy number $N$ and robustness (subplot (b)). This result shown in subplot (a) indicates that three hierarchies lead to the highest strength. For robustness, the data shown in subplot shows that as the number of hierarchical levels is altered, the accessible values for robustness changes as well. For few and high levels of hierarchies, robustness values tend to be high (note that the best performer $\{4096,4\}$ is indicated in the plot). For intermediate levels of hierarchies (between 3 and 8 , approximately) the accessible robustness values range almost from 0 to 1 . The system with highest strength is also indicated in this plot (with a robustness value close to 0) (see also Table 2 for an overview over the performance of various structures). 
superior performance. Biological materials may have achieved these particular structures through evolutionary adaptation of hierarchical structures. It suggests that biology has long implemented the efficient use of hierarchy as a materials "design" variable and established highly adapted geometries for use in filaments, structural materials and for mechanical signaling.

- There exists an optimal hierarchical depth to provide optimal mechanical performance. We find that three level hierarchies are the best performers (Fig. 7(a)) as well as Table 2).

- The introduction of hierarchical degrees of freedom (HDOFs) can serve as a beneficial tool for system description and the analysis of system behavior. Such degrees of freedom can be utilized in the development of present single level models to multi-level hierarchical forms, as illustrated here with the Hierarchical Bell Model.

Clearly there is a relationship between a materials robustness, strength and level of hierarchical complexity, exemplified throughout a range of biological materials and explicitly shown in the current investigation of $\mathrm{AH}$ assemblies and the Hierarchical Bell Model. A possible conceptual explanation is the increase of system entropy with an increase in hierarchies, as discussed through the parameter $\eta$, a measure for the number of HDOFs. By using an approach that multiple hierarchies and HDOFs inherently increase the number of microstates resulting in global system macrostates, the entropy is also increased. The hierarchical structure provides additional complexity to the energy landscape of the system, increasing the total number of possible energy states for a given loading. This can be compared to structural dynamics, where increasing modal degrees of freedom increases possible modal shapes, frequencies of the system, and energy dissipation capacity. Likewise, the number of HDOFs explicitly changes the entropy of the system and affects strength and robustness.

Self-organization phenomena in nature repeatedly demonstrate that spontaneously formed stable static structures attain levels of repeated hierarchical complexity. However, as shown in our analysis, certain combinations of hierarchies produce maximum strength or robustness independent of number of hierarchies (system 4) or maximum redundancies (system 1), as shown for the 8 element system (Fig. 4(b)). This suggests that a simple correlation of energy or entropy and number of hierarchical levels or elements may be misleading. In fact, the strength of each system is proportional to the number of defined HDOFs (for example, the $\{x, 2\}$ family of systems result in both the largest number of HDOFs and the largest strengths). Robustness is not as clearly related to number of HDOFs (robustness increases with increasing number of elements on each scale), but the consistent results of the $\{x, 4\}$ family of systems warrants further investigation (see also Table 2 for a summary of best performance structures). The case study reported here provides some first insight into this complex relationship. Further aspects will be investigated systematically in future work. 
The results shown in Fig. 7 further suggest that the level of hierarchical depth and strength may be balanced in biological protein materials, since the robustness and strength are not completely inversely proportional, allowing biological materials to maximize the mechanical performance while minimizing the use of materials. Overall our analysis illustrates that the introduction of hierarchies is the key to unify disparate material properties. Applying this insight to the design of materials will allow an extended use of hierarchies in bio-inspired or biomimetic synthetic materials at nanoscale, such as hierarchically organized CNT-bundles, nanowires, CNT-protein or polymer-protein composites [Cui et al., 2007; Hule and Pochan, 2007; Winey and Vaia, 2007]. The combination of synthetic and natural constituting elements (e.g. proteins) could be a particularly promising strategy. Even though derived for a different material, our results that the optimal number of hierarchies is three agree with earlier findings for a bioinspired nacre mimicking carbon nanotube material [Pugno, 2006].

The increasing use of protein building blocks in the development of novel nanomaterials, such as nanowires, nanotubes, and others [Scheibel et al., 2003] requires the development of new engineering models that enable the systematic design of the use of nanoscale constituents in the makeup of larger-scale materials. Our model provides such insight and design rules, here shown for the example of AH structures, which facilitate the development of novel nanostructures based on proteins. Most importantly, our model explains a fundamentally new concept, that is, by simply rearranging the same number of nanoscale elements into hierarchies, one can change the performance of the material in the strength-robustness space (see e.g. Fig. 5). Notably this makes the continuous invention of new basic building blocks unnecessary. Thus the broad application of universal building blocks in highly diverse architectures might be a biological strategy that enables adaptation to changes in the environment directly by adopting the structural arrangement of the same basic building blocks [Ackbarow and Buehler, 2008; Buehler and Yung, 2009].

This concept also appears on the level of primary structure: instead of inventing new amino acids, a limited number of 20 amino acids are combined in different ways, which allows creating all biological materials [Buehler and Yung, 2009]. Thus, further studies must be carried out to investigate this hypothesis in greater depth. In additional to the biological context, detailed studies of this concept as an "engineering paradigm" are necessary. By utilizing the introduction of HDOFs, hierarchical materials and such general assemblies of subsystems can be easily described. Additionally, the HDOFs can be defined independently of system information, and are identical throughout a range of structural scales and constituent materials. This might result in synthetic materials as mechanomutable, smart structures, which continuously and independently adapt to environmental changes at each lengthand time-scale, consisting of a handful of building blocks on the nanoscale.

Even though our modeling reported here has been focused on AH protein structures, the main results of the work should be generally valid for other protein 
structures that are primarily stabilized by "weak" H-bonds, in particular the finding that hierarchical arrangements of $\mathrm{H}$-bonds are crucial for the ability of protein materials to combine strength and robustness. This property of biological protein materials has often been pointed out in the literature [Fratzl and Weinkamer, 2007; Fratzl et al., 2004], but has not yet been explained based on a fundamental physics model as accomplished here with the Hierarchical Bell Model (Eqs. (2.12) and (2.13)). The observed behavior provides further evidence that this behavior may have its origin at the molecular scale. This insight could be crucial to translate biological material concepts towards nanotechnology applications. However, caution must be taken since other types of chemical bonding as well as the environmental conditions such as temperature or $\mathrm{pH}$ (possibly strengthening and weakening different bonds) may also contribute to a protein's mechanical stability (see also the discussion in the introduction), and that these models should be considered appropriately. Further, interactions other than H-bonding must be considered, in particular for larger protein assemblies. This includes strong intermolecular interactions due to charged protein surfaces, hydrophobic effects, or strong cross-links (e.g. mediated through disulfide bonds). The inclusion of these effects is left to future work.

The failure of engineering materials and structures has been studied extensively and has impacted our world by enabling the design of complex structures such as buildings, airplanes, cars and devices. However, the mechanisms of failure in biological materials and how it leads to the breakdown of components in our body is not well understood. Thus, characterizing how protein materials fail has significant implications that may eventually lead to an improved understanding of diseases and injuries. The link between molecular structure and material properties in the strength-robustness domain may lead to a paradigm shift in the understanding of which physical mechanisms govern the behavior of biological systems. Understanding the fundamental physical laws that control the properties of hierarchical protein materials enables us also to link the structural protein organization to the appropriate biological functions. Engineering materials using a bottom-up approach that begins at the atomistic level, inspired by biological protein material concepts, may transcend the borders that currently lie between life sciences and engineering. The transfer towards the design of novel nanostructures facilitates the development of de novo multifunctional and mechanically active, tunable and changeable materials, for example new organic and organic-inorganic composites that primarily consist of chemical elements that appear in our environment in practically unlimited amount (chemical elements such as $\mathrm{C}, \mathrm{H}, \mathrm{N}, \mathrm{O}, \mathrm{S}$ ).

This may lay the foundation for a new engineering paradigm that includes the design of structures and materials starting at the molecular level, from bottom up, to the macroscale, to create new materials and structures that mimic and exceed the properties found in biological analogs. The development of a fundamental science driven framework that involves a solid understanding of fundamental concepts is crucial for studies of biological systems, disease diagnosis and treatment, as well 
as the design of novel biomaterials. It is the key to reverse-engineer the human body, the key to understand diseases at multi-scale levels (cancer, genetic diseases, infectious diseases), to enable advanced treatments (intervention at level of relevant proteins, nanomedicine, protein hierarchies, biomaterials) and diagnostics (mechanical disease signature, protein misregulation).

Recently it was reported that the first bundles of coiled-coil proteins were generated synthetically [Papapostolou et al., 2007], and many other peptide synthesis techniques are progressing rapidly [Langer and Tirrell, 2004; Mershin et al., 2005; Papapostolou et al., 2007; Smeenk et al., 2005; Zhao and Zhang, 2006], allowing to create self-assembled nanostructures of amyloid fibers [Scheibel et al., 2003] or collagen fibrils [Kotch and Raines, 2006]. The model reported in this paper, combined with these new manufacturing techniques, may be the first step towards a de novo bottom-up structural design of protein based materials. In addition to protein materials this theory could be applicable to other nanoscale devices, which exhibit a hierarchical structure and are governed by stochastic processes of failure, such as polymer brushes or multilayer films. A detailed analysis of the applicability of this theory to these structures is left to future work.

The field of genomics is concerned with the study of genes and their effects on macroscopic functions, and has led to considerable medical advances. Genomics, however, does not elucidate material properties, nor the mechanistic relation of hierarchical multi-scale structures and their resulting properties. The multi-scale behavior of protein assemblies with the goal of elucidating the relation between structure and material properties represents a grand challenge at the interface of materials science and biology. This gap in understanding can be closed by systematically studying the material properties of hierarchical protein structures and their effect on the macroscopic properties, an approach part of a larger effort to study the role of materials in biology, referred to as materiomics [Buehler and Keten, 2008]. Here we have focused on the strength properties of hierarchical AH protein structures.

\section{Acknowledgements}

This research was supported by the Army Research Office, grant \# W911NF-061-0291 (program officer Dr. Bruce LaMattina), by a National Science Foundation CAREER Award (program manager Dr. Jimmy Hsia), grant \# 0642545, and by a grant from the Air Force Office of Scientific Research, grant \# FA9550-08-1-0321 (program manager Dr. Les Lee). This work was supported in part by the MRSEC Program of the National Science Foundation under award number DMR-0819762. TA acknowledges support from the German National Academic Foundation (Studienstiftung des Deutschen Volkes) and the Hamburg Foundation for research studies abroad (Hamburger Stipendienprogramm). We thank Professor Lothar Gaul (University Stuttgart) and Professor Reinhard Lipowsky (Max-Planck Institute of Colloids and Interfaces Potsdam) for their continuous interest and support of our work. 


\section{References}

Ackbarow, T. and Buehler, M. J. [2007] "Superelasticity, energy dissipation and strain hardening of vimentin coiled-coil intermediate filaments: Atomistic and continuum studies," Journal of Materials Science 42(21), 8771-8787.

Ackbarow, T. and Buehler, M. J. [2008] "Hierarchical coexistence of universality and diversity controls robustness and multi-functionality in protein materials," Theoretical and Computational Nanoscience 5(7), 1193-1204.

Ackbarow, T. and Buehler, M. J. [2009] "Alpha-helical protein domains unify strength and robustness through hierarchical nanostructures," Nanotechnology 20, 075103.

Ackbarow, T., Chen, X., Keten, S. and Buehler, M. J. [2007] "Hierarchies, multiple energy barriers and robustness govern the fracture mechanics of alpha-helical and beta-sheet protein domains," P. Natl. Acad. Sci. USA 104, 16410-16415.

Ahl, V. and Allen, T. F. H. [1996] Hierarchy Theory - A Vision, Vocabulary, and Epistemology (New York: Columbia University Press).

Alberts, B., Johnson, A. Lewis, J., Raff, M., Roberts, K. and Walter, P. [2002] Molecular Biology of the Cell (Taylor \& Francis).

Ashby, M. F., Gibson, L.J., Wegst, U. and Olive, R. [1995] "The Mechanical Properties of Natural Materials. I. Material Property Charts," Proceedings: Mathematical and Physical Sciences 450(1938), 123-140.

Bell, G. I. [1978] "Models for specific adhesion of cells to cells," Science 200(4342), 618627.

Buehler, M. J. and Ackbarow, T. [2007] "Fracture mechanics of protein materials," Materials Today 10(9), 46-58.

Buehler, M. J. and Gao, H. [2006] "Dynamical fracture instabilities due to local hyperelasticity at crack tips," Nature 439, 307-310.

Buehler, M. J. and Keten, S. [2008] "Elasticity, strength and resilience: A comparative study on mechanical signatures of $\alpha$-helix, $\beta$-sheet and tropocollagen domains," Nano Research 1(1), 63-71.

Buehler, M. J. and Yung, Y. C. [2009] "Deformation and failure of protein materials in physiologically extreme conditions and disease," Nature Materials 8(3), 175-188.

Buehler, M.J., Keten, S. and Ackbarow, T. [2008] "Theoretical and computational hierarchical nanomechanics of protein materials: Deformation and fracture," Progress in Materials Science 53, 1101-1241.

Chopra, A. K. [2007] Dynamics of Structures, Theory and Applications to Earthquake Engineering, 3rd Edn. (Pearson Prentice Hall).

Cui, X. Q., Li, C. M., Zang, J. F., Zhou, Q., Gan, Y., Bao, H. F., Guo, J., Lee, V. S. and Moochhala, S. M. [2007] "Biocatalytic generation of ppy-enzyme-CNT nanocomposite: From network assembly to film growth," Journal of Physical Chemistry C 111(5), 2025-2031.

Dudko, O. K., Hummer, G. and Szabo, A. [2006] "Intrinsic rates and activation free energies from single-molecule pulling experiments," Physical Review Letters 96(10), 108101.

Evans, E. and Ritchie, K. [1997] "Dynamic strength of molecular adhesion bonds," Biophysical Journal 72(4), 1541-1555.

Fratzl, P. and Weinkamer, R. [2007] "Nature's hierarchical materials," Progress in Materials Science 52, 1263-1334.

Fratzl, P., Gupta, H. S., Paschalis E. P. and Roschger, P. [2004] "Structure and mechanical quality of the collagen-mineral nano-composite in bone," Journal of Materials Chemistry 14(14), 2115-2123. 
Herrmann, H. and Aebi, U. [2004] "Intermediate filaments: Molecular structure, assembly mechanism, and integration into functionally distinct intracellular scaffolds," Annual Review of Biochemistry 73, 749-789.

Hirth, J. P. and Lothe, J. [1982] Theory of Dislocations (Wiley-Interscience).

Hule, R. A. and Pochan, D. J. [2007] "Polymer nanocomposites for biomedical application," MRS Bulletin 32(4), 354-358.

Ingber, D. E. [2006] "Cellular mechanotransduction: Putting all the pieces together again," FASEB Journal 20(7), 811-827.

Keten, S. and Buehler, M. J. [2008a] "Asymptotic strength limit of hydrogen-bond assemblies in proteins at vanishing pulling rates," Physical Review Letters 100(19): 198301.

Keten, S. and Buehler, M. J. [2008b] "Geometric confinement governs the rupture strength of h-bond assemblies at a critical length scale," Nano Letters 8(2), 743-748.

Kitano, H. [2002] "Computational systems biology," Nature 420(6912), 206-210.

Kotch, F. W. and Raines, R. T. [2006] "Self-assembly of synthetic collagen triple helices," Proceedings of the National Academy of Sciences of the United States of America 103(9), 3028-3033.

Langer, R. and Tirrell, D. A. [2004] "Designing materials for biology and medicine," Nature 428 (6982), 487-492.

Marszalek, P. E., Lu, H., Li, H. B., Carrion-Vazquez, M., Oberhauser, A. F., Schulten, K. and Fernandez, J. M. [1999] "Mechanical unfolding intermediates in titin modules," Nature 402(6757), 100-103.

Mershin, A., Cook, B., Kaiser, L. and Zhang, S. G. [2005] "A classic assembly of nanobiomaterials," Nature Biotechnology 23(11), 1379-1380.

Murphy, K. P., ed. [2001] Protein Structure, Stability, and Folding, Methods in Molecular Biology, ed. N. W. John. Vol. 168, Humana Press: Totowa.

Papapostolou, D., Smith, A. M., Atkins, E. D. T., Oliver, S. J., Ryadnov, M. G., Serpell, L. C. and Woolfson, D. N. [2007] "Engineering nanoscale order into a designed protein fiber," PNAS, p. 0700801104.

Pugno, N. [2006] "Mimicking nacre with super-nanotubes for producing optimized supercomposites," Nanotechnology 17, 5480-5484.

Qin, Z., Feng, X. Q., Zou, J., Yin, Y. J. and Yu, S. W. [2007] "Superior flexibility of super carbon nanotubes: Molecular dynamics simulations," Applied Physics Letters 91:043108.

Rowat, A. C., Lammerding, J. Herrmann, H. and Aebi, U. [2008] "Towards an integrated understanding of the structure and mechanics of the cell nucleus," Bioessays 30(3), $226-36$

Scheibel, T., Parthasarathy, R., Sawicki, G., Lin, X.-M., Jaeger, H. and Lindquist, S. L. [2003] "Conducting nanowires built by controlled self-assembly of amyloid fibers and selective metal deposition," Proceedings of the National Academy of Sciences of the United States of America 100(8), 4527-4532.

Seifert, U., [2000] "Rupture of multiple parallel molecular bonds under dynamic loading," Physical Review Letters 84(12), 2750-2753.

Sheu, S.-Y., Yang, D.-Y., Selzle, H. L. and Schlag, E. W. [2003a] "Energetics of hydrogen bonds in peptides," PNAS 100(22), 12683-12687.

Sheu, S. Y., Yang, D. Y., Selzle, H. L. and Schlag, E. W. [2003b] "Energetics of hydrogen bonds in peptides," Proceedings of the National Academy of Sciences of the United States of America 100(22), 12683-12687.

Smeenk, J. M., Otten, M. B. J., Thies, J., Tirrell, D. A., Stunnenberg, H. G. and van Hest, J. C. M. [2005] "Controlled assembly of macromolecular beta-sheet fibrils," Angewandte Chemie-International Edition 44(13), 1968-1971. 
112 Z. Qin et al.

Sotomayor, M. and Schulten, K. [2007] "Single-molecule experiments in vitro and in silico," Science 316(5828), 1144-1148.

Winey, K. I. and Vaia, R. A. [2007] "Polymer nanocomposites," MRS Bulletin 32(4), 5.

Zhao, X. J. and Zhang, S. G. [2006] "Molecular designer self-assembling peptides," Chemical Society Reviews 35(11), 1105-1110. 